OPEN ACCESS

Edited by:

Huai L. Feng,

Weill Cornell Medical Center,

United States

Reviewed by:

Borut Kovacic,

Maribor University Medical Centre,

Slovenia

Linli Hu,

Zhengzhou University, China

${ }^{*}$ Correspondence:

Shi-Bin Chao

chaoshibin@163.com

Specialty section:

This article was submitted to

Reproduction,

a section of the journal

Frontiers in Endocrinology

Received: 24 June 2021

Accepted: 25 August 2021

Published: 20 September 2021

Citation:

Li J-C, Wang Y-H, Peng I-Y,

Zhou $Y$ and Chao S-B (2021)

A Novel Promising Endometrial

Preparation Protocol for

Frozen-Thawed Embryo

Transfer: A Randomized

Controlled Trial.

Front. Endocrinol. 12:730059. doi: 10.3389/fendo.2021.730059

\section{A Novel Promising Endometrial Preparation Protocol for Frozen- Thawed Embryo Transfer: A Randomized Controlled Trial}

\author{
Jian-Chun $\mathrm{Li}^{1,2}$, Yan-Hong Wang ${ }^{2}$, li-Ying Peng ${ }^{3}$, Yun Zhou ${ }^{2}$ and Shi-Bin Chao ${ }^{2,4^{*}}$ \\ ${ }^{1}$ Department of Clinical Laboratory, The First Affiliated Hospital of NanChang University, NanChang, China, ${ }^{2}$ ART Center, \\ Maternal and Child Health Care Hospital, ShangRao, China, ${ }^{3}$ Department of Reproductive Medicine, Reproductive Hospital \\ Affiliated to Jiangxi University of Traditional Chinese Medicine, Nanchang, China, ${ }^{4}$ Department of Obstetrics and Gynecology, \\ Fuzhou Medical College of NanChang University, Fuzhou, China
}

Background: In recent years frozen-thawed embryo transfer (FET) has played an increasingly important role in ART, but there is limited consensus on the most effective method of endometrial preparation (EP) for FET. Inspired by significantly higher implantation rate and clinical pregnancy rate of the depot $\mathrm{GnRH}$-a protocol, we proposed a novel EP protocol named down-regulation ovulation-induction (DROI) aimed to improve pregnancy outcomes of FET.

Methods: This was a single-center, randomized controlled pilot trial. A total of 307 patients with freeze-all strategy scheduled for first FET were enrolled in the study. A total 261 embryos were transferred in DROI-FET group including 156 patients and 266 embryos were transferred in mNC-FET group including 151 patients. Reproductive outcomes were compared between the two groups.

Results: The basic characteristics of patients, and the average number, quality and stage of embryos transferred were comparable between the two groups. Our primary outcome, implantation rate(IR) in DROI-FET group, was significantly higher than that of the $\mathrm{mNC}$ FET group (54.41\% versus $35.71 \%, \mathrm{P}<0.01)$. The clinical pregnancy rate (CPR) and ongoing pregnancy rate (OPR) in DROI-FET group was also higher than that in mNC-FET group (69.87\% versus 50.33\%, $\mathrm{P}<0.01 ; 64.10 \%$ versus $42.38 \%, \mathrm{P}<0.01$ ).

Conclusion(s): Compared to existing endometrial preparation methods, the DROI protocol might be the more efficient and promising protocol.

Keywords: frozen-thawed embryo transfer, endometrial preparation, endometrial receptivity, embryo implantation, in vitro fertilization, down regulation ovulation induction

\section{INTRODUCTION}

With the application of vitrification techniques and widespread ongoing adoption of freeze-all strategy, the number of FET cycles has dramatically increased (1). Whether in fresh ET or in FET, embryo implantation relies upon embryo quality, endometrial receptivity, and synchronization between embryo and endometrium (2). From the beginning of IVF, embryo quality has been the 
most emphasized factor (3), while more and more studies are paying attention to endometrial receptivity (4). But there is no consensus on either the explicit mechanism or the solution to improve endometrial receptivity up to now $(5,6)$.

FET cycle overcomes some adverse effects of fresh ET, such as reducing the risk of ovarian hyperstimulation syndrome (OHSS) and avoiding some negative effects on the endometrium. FET resulted in higher clinical pregnancy rate than did fresh ET $(2,7)$. This has fuelled the call for a new strategy called freeze-all strategy where no fresh embryo transfer is conducted and all available embryos are cryopreserved, and transferred in subsequent FET cycles $(2,8)$.

FET protocols are relatively simple in the attempt to prepare the endometrium (9). Various endometrial preparation regimens have been developed for FET. The most commonly applied protocols include natural cycle FET (NC-FET), modified NCFET using HCG, hormone replacement therapy (HRT) cycle with or without $\mathrm{GnRH}$ agonists, and ovulation induction cycle. Nevertheless, by far, there is no superiority of any regimen for EP over another one in terms of reproductive outcomes $(9,10)$.

Recently, several studies reported that depot GnRH-a COS protocol yields relatively high CPR and IR in fresh ET $(5,11,12)$. Lei J reported that IR per fresh ET cycle were 51\% in the depot GnRH-a protocol, 37\% in the GnRH antagonist, and $45 \%$ in the long GnRH-a protocols, respectively $(\mathrm{P}<0.01)(12)$. The higher IR is considered to be through the way of improving endometrial receptivity, because the outcomes of the embryos derived from the COS cycles might not be superior to those from GnRH antagonist cycles in subsequent FET cycles $(11,13,14)$. The latest research published in June 2020 elucidated the mechanism by which depot $\mathrm{GnRH}$-a protocol improves endometrial receptivity. The protocol enhanced several well-established biomarkers for endometrial receptivity such as HOXA10, MEIS1 and LIF gene expression and thereby helps to improve endometrial receptivity (12).

Based on the suggestion that depot GnRH-a protocol might improve endometrial receptivity, we proposed a novel EP protocol named down-regulation ovulation -induction (DROI). The purpose of the present study was to testify whether the novel protocol could improve IR, CPR and OPR compared to modified nature cycle (mNC) EP protocol.

\section{MATERIALS AND METHODS}

\section{Study Design and Participants}

This is a single-centre RCT performed at the ART centre of ShangRao Maternal and Child Health Care Hospital, China. The minimal sample size calculated with a significance level of 0.05 and with statistical power of $80 \%$ is 973 for each group (15). Ideally, sufficient sample size would have been recruited to this research to determine equivalence between the two EP protocols. So this study can thus only be viewed as a pilot RCT study.

The study conformed to the 'Declaration of Helsinki for Medical Research involving Human Subjects' and was approved by the hospital's ethics committee. Infertile women undergoing first FET cycles in the period from March 2018 to May 2020 were enrolled. This RCT trial was registered at the Chinese Clinical Trial Registry, number ChiCTR2000039804. The candidate patients obtained detailed information of both protocols, including the duration of the down-regulation and the potential risk of pituitary suppression. All patients enrolled gave written informed consent for the procedures.

A total of 360 patients with freeze-all strategy scheduled for first FET were randomly assigned to two study groups in a 1:1 ratio. Participants in the RCT Random allocation was performed by a study doctor at endometrial preparation by means of computer generated random numbers in sealed, unlabelled envelopes. Doctors, patients and nurses administering the interventions were not blinded to the treatment assigned. Inclusion criteria were age $<38$ years, normal menstrual cycle, BMI 18-28 kg/m2, and basal FSH level $<10 \mathrm{IU} / \mathrm{ml}$. Patients diagnosed with poor ovarian response (antral follicle count (AFC) $<7$ follicles or anti-Müllerian hormone $(\mathrm{AMH})<1.1 \mathrm{ng} /$ $\mathrm{ml}$ ), hyperprolactinemia, endometriosis, hydrosalpinx and uterine abnormalities, thyroid disease were excluded.

\section{COS and Embryo Vitrification, Thawing, and Transfer}

All participants were given GnRH antagonist regimen or Progestin-primed ovarian stimulation (PPOS) for ovarian stimulation as extensively described elsewhere $(2,16)$. When at least two follicles were $18 \mathrm{~mm}$ or greater in mean diameter, human chorionic gonadotropin (HCG) at a dose of 4000-10000 IU or $0.2 \mathrm{mg}$ of triptorelin was administered to induce the final maturation of oocytes. Oocyte retrieval was performed 34-36 h after trigger. Embryo morphology was assessed and graded on Day 3 according to the Cummins criteria (17). Generally, two Grade I or Grade II embryos were vitrified on Day 3, and supernumerary embryos were cultured continuously until the blastocyst stage on Day 5 or Day 6 before vitrification. The vitrification procedure was performed following standard protocols using Kitazato Freeze Kit (Kitazato Corporation, Janpan). Embryos containing $\geq 7$ cells, with $\leq 20 \%$ fragmentation and symmetric or slightly asymmetric blastomere distribution, but without multinucleation on Day 3 and blastocysts graded $\geqq 4 \mathrm{BB}$ according to Gardner morphological criteria were classified as top-quality embryos (2).

\section{Endometrial Preparation Before Embryo Transfer}

In the DROI-FET group, pituitary down-regulation was achieved by full dose (3.75 mg) of Leuprorelin Acetate (Lizhu Pharmaceutical Trading Co, Shanghai, China) at day 2 or day 3 of the menstrual cycle. Gonadotropin stimulation started after 35-42 days when the biggest antral follicle was larger than 5-6 $\mathrm{mm}$. Patients received 75-150 IU of HMG injection (Lizhu Pharmaceutical Trading Co, ZhuHai, China) daily according to $\mathrm{AMH}, \mathrm{AFC}$, especially ovarian response in the COS cycles. The specific starting GN dose was that when $\geq 10$ oocytes were harvested in the fresh cycle, the starting dose was about half of the COS starting dose, and when $<10$ oocytes, the starting dose 
was about two-thirds of the COS starting dose. Gonadotropin stimulation was adjusted according to transvaginal ultrasound and serum E2, LH and P levels. Gonadotropin stimulation continued until endometrial thickness $\geq 7 \mathrm{~mm}$ and meeting one of the following two criteria: (1) If there were leading follicles, the number of these leading follicles which had a mean diameter of $\geq 16 \mathrm{~mm}$ was between $1-3$, with serum estradiol level $200-800 \mathrm{ng} / \mathrm{l}$ and progesterone level $<1.5 \mathrm{ng} / \mathrm{mL}$; (2) If there was no dominant follicle $\geq 16 \mathrm{~mm}$, at most four follicles reached the diameter between 12 and $15 \mathrm{~mm}$, with serum estradiol levels between 150 and 1000ng/l and progesterone level $<1.5 \mathrm{ng} / \mathrm{mL}$. A dose of HCG 5000-10000 IU (Lizhu Pharmaceutical Trading Co.) was injected at 9:00 p.m. and ET was arranged 5 days later for D3 embryos or 7 days later for blastocysts. Progesterone supplement was initiated 2 days after ovulatory trigger as fresh ET described elsewhere (6). Procedures of DROI-FET protocol is summarized in Figure 1.

Patients undergoing modified NC-FET (mNC-FET) attended for ultrasound evaluation between days 10 and 12 of their menstrual cycle to confirm follicular growth and endometrial thickness. Participants commenced self-monitoring of ovulation by using urinary dipsticks. Ultrasound monitoring continued until the dominant follicle reached $16-18 \mathrm{~mm}$ and endometrial thickness $\geq 7$ mm. A dose of HCG 5000-10000 IU (Lizhu Pharmaceutical Trading Co) was given subcutaneously to trigger ovulation. In both DROIFET and mNC-FET groups, luteal support was continued to 10 weeks of gestation if a pregnancy occurred.

\section{Outcome Parameters and Statistical Analysis}

The primary outcome of the study was implantation rate. The secondary end points included clinical pregnancy rate and ongoing pregnancy rate.
Continuous data were compared with the Student t test and categoric variables were compared with the $\chi 2$ test. Multivariable logistic regression analysis was used to evaluate the possible relationship between the protocol of endometrial preparation and CPR, OPR after adjusting for confounding factors, including age, BMI, BFSH, infertility duration, endometrium thickness, number of embryos transferred, and embryo developmental stage. All statistical analyses were performed by using the Statistical Package for Social Sciences (SPSS) version 22.0. A $\mathrm{P}$ value $<0.05$ was considered to be statistically significant.

\section{RESULTS}

\section{Study Population}

A total of 360 women were randomized and 307 had ET and completed the study. Reasons for dropout are summarized in Figure 2. Remaining patients received treatment according to study group allocation, resulting in 156 patients $(50.81 \%)$ receiving DROI-FET and 151 (49.19\%) receiving $\mathrm{mNC}-\mathrm{FET}$.

\section{Baseline Characteristics}

Patients' baseline characteristics are detailed in Table 1. No significant difference was observed between the two treatment groups regarding age, BMI, duration of infertility, total antral follicle count, indication for IVF, or type of infertility. The baseline hormone profile and COS protocol in fresh cycles were similar between the two groups.

\section{Cycle Characteristics of FET}

The quantity, quality and developmental stage of embryos are the key factors that affect the outcome of FET. As presented in Table 2, the average number of embryos transferred in the

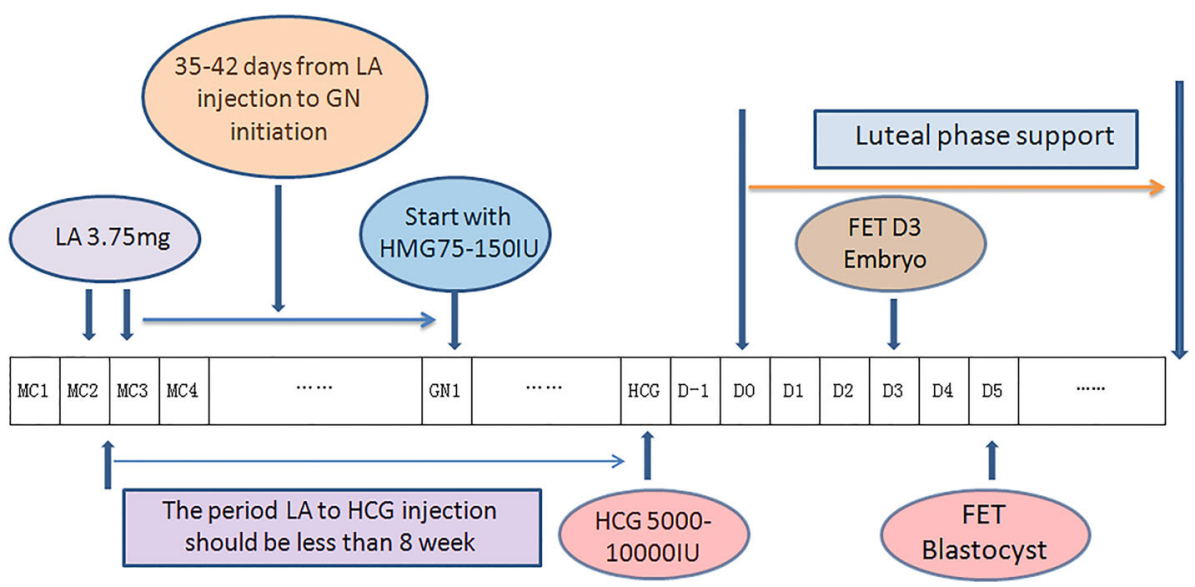

FIGURE 1 | Flow chart of down-regulation ovulation-induction procedure. One depot of 3.75 mg leuprorelin acetate was injected at the 2nd or 3rd day of the menstrual cycle, in which ovarian stimulation with 75-150 IU Gns would start 35-42 days later along with confirmation of pituitary downregulation. Gonadotropin stimulation continued until endometrial thickness $\geq 7 \mathrm{~mm}$. A dose of HCG 5000-1000 IU was injected at 9:00 p.m. and ET was arranged 5 days later for D3 embryos or 7 days later for blastocysts. Progesterone supplement was initiated 2 days after ovulatory trigger as fresh ET. (LA, leuprorelin acetate; GN, Gonadotropin; HCG, Human Chorionic Gonadotropin; MC, Mnstrual Cycle; D0, theoretical oocyte retrieval day). 


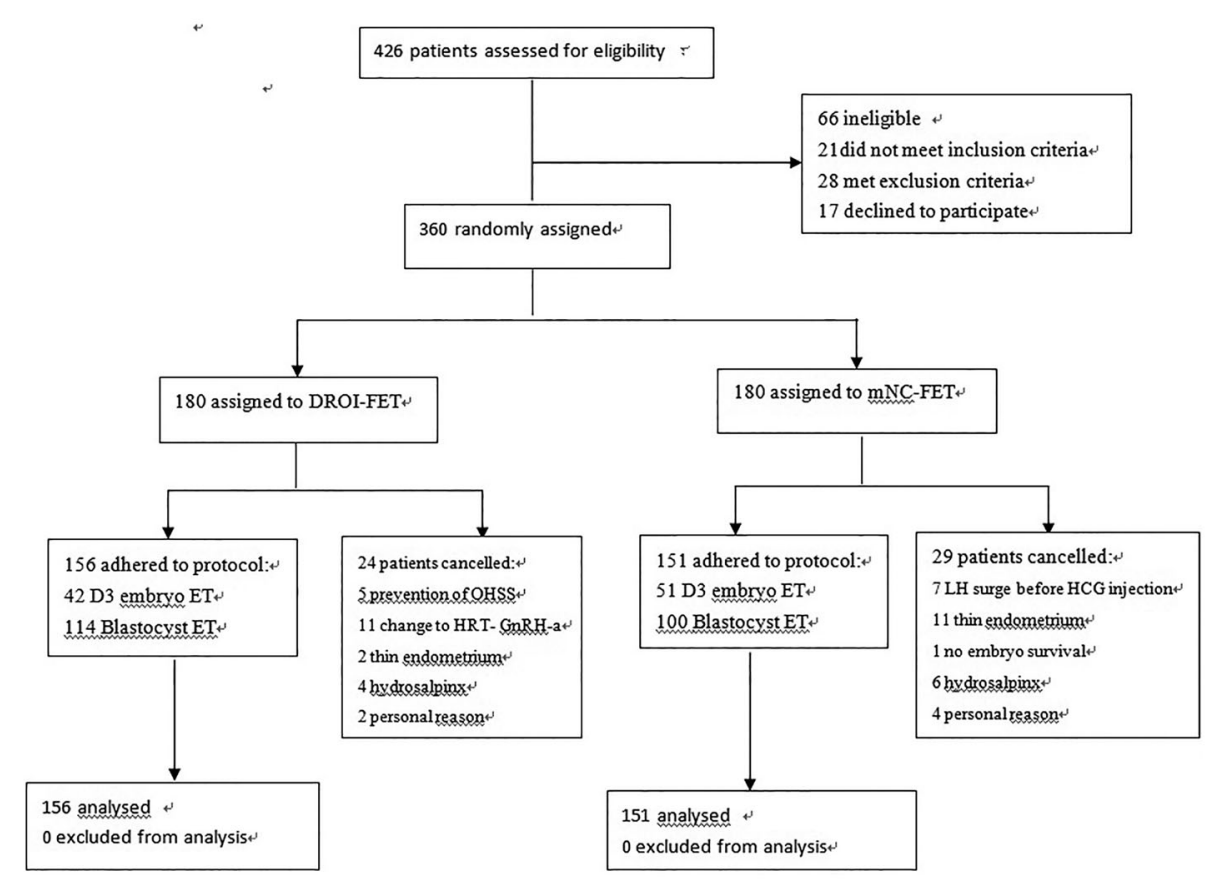

FIGURE 2 | Flowchart showing of enrollment and randomization of study patients.

TABLE 1 | Basic characteristics of patients at the cycle level.

\begin{tabular}{|c|c|c|c|}
\hline Characteristic & DROI-FET (156) & mNC-FET (151) & $P$ value \\
\hline Age (y) & $30.58 \pm 4.43$ & $30.50 \pm 4.35$ & 0.87 \\
\hline Body mass index $(\mathrm{kg} / \mathrm{m} 2)$ & $21.27 \pm 2.55$ & $21.50 \pm 2.35$ & 0.41 \\
\hline Infertility duration (years) & $3.33 \pm 1.25$ & $3.30 \pm 1.43$ & 0.84 \\
\hline \multicolumn{4}{|l|}{ Type of infertility } \\
\hline Primary & $72(46.15 \%)$ & $71(47.02 \%)$ & \\
\hline Secondary & $84(53.85 \%)$ & $80(52.98 \%)$ & 0.88 \\
\hline \multicolumn{4}{|l|}{ Indications for IVF } \\
\hline Tubal factor & $101(64.74 \%)$ & 83(54.97\%) & \\
\hline Male factor & 30(19.23\%) & $28(18.54 \%)$ & \\
\hline Unexplained infertility & 6(3.84\%) & $5(3.31 \%)$ & \\
\hline Others & $19(12.18 \%)$ & $35(23.18 \% \%)$ & 0.08 \\
\hline \multicolumn{4}{|l|}{ Baseline sex hormone } \\
\hline FSH (IU/L) & $6.57 \pm 2.21$ & $6.59 \pm 2.09$ & 0.94 \\
\hline LH (IU/L) & $5.06 \pm 2.21$ & $5.19 \pm 2.29$ & 0.61 \\
\hline E2 (pg/mL) & $32.95 \pm 14.15$ & $33.00 \pm 15.27$ & 0.98 \\
\hline$P(n g / m L)$ & $0.36 \pm 0.25$ & $0.34 \pm 0.25$ & 0.61 \\
\hline Total antral follicle count & $12.83 \pm 5.03$ & $13.73 \pm 4.34$ & 0.94 \\
\hline \multicolumn{4}{|l|}{ Protocol for COS } \\
\hline GnRH-ANT & $101(64.74 \%)$ & $86(56.95 \%)$ & \\
\hline PPOS & $55(35.26 \%)$ & $65(53.05 \%)$ & 0.16 \\
\hline
\end{tabular}

Data are presented as mean $\pm S D$ for continuous variables and $n$ (\%) for dichotomous variables. All $P$ values were assessed with the use of $\chi 2$ or Student $t$ test. DROI, down-regulation ovulation-induction; $\mathrm{mNC}$, modified nature cycle; FET, frozen-thawed embryo transfer.

DROI-FET group was a little less than that in the mNC-FET group, but there was no significance (1.67 \pm 0.47 VS $1.76 \pm 0.43$, $\mathrm{p}>0.05$ ), and the number of double embryo transfers was higher in the mNC-FET group and neared significance with $p=0.09$. The proportions of patients with single embryo transfer, two-embryo transfer, transferred embryo stage at cleavage or blastocyst stage,
TABLE 2 | Cycle characteristics at transfer level.

\begin{tabular}{|c|c|c|c|}
\hline Characteristic & $\begin{array}{l}\text { DROI-FET } \\
\text { (156) }\end{array}$ & mNC-FET (151) & $\begin{array}{c}\mathbf{p} \\
\text { value }\end{array}$ \\
\hline $\begin{array}{l}\text { Average number of embryos } \\
\text { transferred }\end{array}$ & $1.67 \pm 0.47$ & $1.76 \pm 0.43$ & 0.08 \\
\hline \multicolumn{4}{|l|}{ Number of embryos transferred } \\
\hline 1 & $51(32.69 \%)$ & $36(23.84 \%)$ & 0.09 \\
\hline 2 & $105(67.31 \%)$ & $115(76.16 \%)$ & \\
\hline TOP-quality embryo transferred & & & 0.08 \\
\hline 0 & $7(4.49 \%)$ & 10(6.62\%) & \\
\hline 1 & $70(44.87 \%)$ & $49(32.45 \%)$ & \\
\hline 2 & $79(50.64 \%)$ & $92(60.93 \%)$ & \\
\hline Embryo stage & & & 0.19 \\
\hline Cleavage stage (day 3 ) & $42(36.84 \%)$ & $51(33.77 \%)$ & \\
\hline Blastocyst (day 5) & $114(63.16 \%)$ & $100(66.23 \%)$ & \\
\hline $\begin{array}{l}\text { Endometrium Thickness on the } \\
\text { day of HCG }(\mathrm{mm})\end{array}$ & $10.62 \pm 1.43$ & $10.51 \pm 1.87$ & 0.57 \\
\hline
\end{tabular}

and top-quality embryo transferred including cleavage stage and blastocyst stage between the two groups were comparable.

\section{Reproductive Outcomes of FET}

Main reproductive outcomes of FET are presented in Table 3. Our primary outcome, implantation rate per embryo transferred in the DROI-FET group, was statistically higher than that in the mNCFET group $(54.41 \%$ versus $35.71 \%, \mathrm{P}<0.01)$. The rate of clinical pregnancy and ongoing pregnancy in the DROI-FET group was also higher than that in the mNC-FET group $(69.87 \%$ versus $50.33 \%, \mathrm{P}<0.01 ; 64.10 \%$ versus $42.38 \%, \mathrm{P}<0.01)$. 76 of 156 women 
in the DROI-FET group had singleton pregnancy, which was similar to that in the mNC-FET group (57 of 151, 37.75\%). The rates of twin pregnancies were higher in DROI-FET group $(21.15 \%$ versus $12.58 \%$; $\mathrm{p}<0.05)$. There was 1 case of intrauterine and ectopic pregnancy in the mNC-FET group and this patient had been transferred two top-quality cleavage embryos.

After adjustment for the above-mentioned confounding factors (Table 4), the CPR remained consistently higher following DROI-FET (adjusted odds ratio [aOR] 2.73, 95\% confidence interval [CI] 1.63-4.58). Furthermore, the OPR was significantly higher in DROI-FET compared with mNC-FET (aOR 2.59, 95\% CI 1.58-4.26) after correcting for confounders.

\section{DISCUSSION}

Since the first success of FET in 1983 (18), it has been widely used to increase the cumulative pregnancy rate without repeated oocyte retrieval procedure. FET contributes to elective single embryo transfer (eSET), avoiding multiple gestations (19), and it is a strategy to prevent OHSS or to cancel embryo transfer if the endometrial environment is not optimal for fresh ET (20). In recent years the number of FET cycles performed has increased dramatically due to the trend towards transferring fewer embryos after a fresh IVF cycle and freeze-all strategy widely accepted (10, 20). Endometrial preparation is the most critical step for FET. The options range from natural cycle FET, over ovarian stimulation, to HRT with GnRH or without GnRH pretreatment. Indeed, in the latest Cochrane review $(9,10)$, based on RCTs comparing different $\mathrm{EP}$ regimens for FET, it was concluded that no regimen was superior to another.

TABLE 3 | Reproductive Outcomes.

\begin{tabular}{lccc}
\hline Characteristic & DROI-FET (156) & mNC-FET (151) & $\begin{array}{c}\mathbf{p} \\
\text { value }\end{array}$ \\
\hline Implantation rate & $142 / 261(54.41 \%)$ & $95 / 266(35.71 \%)$ & $<0.01$ \\
Clinical pregnancy rate & $109 / 156(69.87 \%)$ & $76 / 151(50.33 \%)$ & $<0.01$ \\
Ongoing pregnancy rate & $99 / 156(64.10 \%)$ & $64 / 151(42.38 \%)$ & $<0.01$ \\
Singletons & $76 / 156(48.72 \%)$ & $57 / 151(37.75 \%)$ & 0.05 \\
Twins & $33 / 156(21.15 \%)$ & $19 / 151(12.58 \%)$ & $<0.05$ \\
$\begin{array}{l}\text { Ectopic pregnancy } \\
\text { intrauterine and ectopic }\end{array}$ & 0 & 0 & \\
pregnancy & 0 & 1 & \\
Moderate or severe OHSS & 0 & 0 & \\
& & & \\
\end{tabular}

Data are presented as $n$ (\%) for dichotomous variables. All $P$ values were assessed with the use of $\chi 2$. Other abbreviations as in Table 1.

TABLE 4 | Unadjusted and adjusted odds ratios (ORs) of reproductive outcomes following DORI-FET versus mNC-FET.

\begin{tabular}{lcc}
\hline Outcome & $\begin{array}{c}\text { Unadjusted OR } \\
\mathbf{( 9 5 \% ~} \mathbf{~ C l})\end{array}$ & $\begin{array}{c}\text { Adjusted OR } \\
\mathbf{( 9 5 \% ~} \mathbf{~ I )}\end{array}$ \\
\hline Clinical pregnancy & 2.29 & 2.73 \\
Ongoing pregnancy & $(1.43-3.65)$ & $(1.63-4.58)$ \\
& 2.36 & 2.59 \\
& $(1.49-3.74)$ & $(1.58-4.26)$ \\
\hline
\end{tabular}

Analyses were adjusted for age, body mass index, infertility duration, BMI,bFSH, endometrium thickness, number of embryos transferred, and embryo developmental stage. Cl (confidence interval); Other abbreviations as in Table 1.
Among the three key factors that determine embryo implantation, endometrial receptivity is difficult to evaluate accurately because its mechanism is unclear and there are no objective evaluation criteria. The result of endometrial receptivity is embryo implantation, in return, embryo implantation rate reflects endometrial receptivity. Many studies and efforts have been made to improve endometrial receptivity but there is little consensus $(21,22)$. However, it has been reported that $\mathrm{CPR}$ and IR increase when patients are given a period of artificial amenorrhea caused by GnRH-a $(23,24)$. Depot GnRH-a protocol has been widely used in China and it dramatically increases the CPR and IR in fresh ET $(5,6,12)$. The success of the protocol is thought to be due to improved endometrial receptivity instead of embryo quality because the embryos derived from the protocol have no advantage over those from other COS protocols in FET cycles (25-27). Therefore, we proposed the DROI EP protocol derived from the depot GnRH-a COS protocol to take advantage of the favorable endometrial receptivity.

As expected, the data in the present study showed that IR in the DROI-FET group increased significantly compared with the mNC-FET group. The novel EP method (DROI) mimics the depot GnRH-a procedure but provides a more favorable endocrine environment instead of the supra-physiological level which might lead to endometrium gene expression and structural abnormalities (28).

The only risk of the DROI protocol may be a possibility of OHSS. It should be kept in mind that the DROI was proposed as an EP protocol for FET. So the principle is that as long as the endometrial thickness is up to $7 \mathrm{~mm}$, estrogen is up to $150-200 \mathrm{pg} / \mathrm{l}$, HCG could be injected to prevent risk of OHSS. In our study, there was no case with moderate or severe OHSS in both groups.

The strength of this study is that we propose a novel and promising endometrial preparation protocol completely different from existing EP protocols. However, there are limitations in this study. First, it is a small sample size, single-centre pilot study which may be subject to selection bias. Second, miscarriage rate and live birth rate are not yet presented in the study. Larger sample size and a more detailed study are very much needed to verify the practicability of the protocol in future studies.

\section{CONCLUSION}

Inspired by the depot GnRH-a COS protocol, we proposed a novel endometrial preparation protocol named DROI for FET. Moreover, our RCT data showed that the DROI increased CPR, OPR and especially IR dramatically compared to the $\mathrm{mNC}$. The effects might be associated with improving endometrial receptivity. Given the fact that there is little consensus on the best protocol for existing EP protocol, the DROI might be the more efficient and promising protocol for FET.

\section{DATA AVAILABILITY STATEMENT}

The raw data supporting the conclusions of this article will be made available by the authors, without undue reservation. 


\section{ETHICS STATEMENT}

The studies involving human participants were reviewed and approved by maternal and child health care hospital's ethics committee. The patients/participants provided their written informed consent to participate in this study.

\section{AUTHOR CONTRIBUTIONS}

Conceptualization: J-CL and S-BC. Data curation: Y-HW and YZ. Formal analysis: S-BC. Methodology: S-BC and Y-HW.

\section{REFERENCES}

1. Zhang J, Liu H, Wang Y, Mao X, Chen Q, Fan Y, et al. Letrozole Use During Frozen Embryo Transfer Cycles in Women With Polycystic Ovary Syndrome. Fertil Steril (2019) 112(2):371-7. doi: 10.1016/j.fertnstert.2019.04.014

2. Wei D, Liu JY, Sun Y, Shi Y, Zhang B, Liu JQ, et al. Frozen Versus Fresh Single Blastocyst Transfer in Ovulatory Women: A Multicentre, Randomised Controlled Trial. Lancet (2019) 393(10178):1310-8. doi: 10.1016/S0140-6736(18)32843-5

3. Braude P. Selecting the 'Best' Embryos: Prospects for Improvement. Reprod BioMed Online (2013) 27(6):644-53. doi: 10.1016/j.rbmo.2013.08.009

4. Casper RF. It's Time to Pay Attention to the Endometrium. Fertil Steril (2011) 96(3):519-21. doi: 10.1016/j.fertnstert.2011.07.1096

5. Ying Y, Yang T, Zhang H, Liu C, Zhao J. Prolonged Pituitary DownRegulation With Full-Dose of Gonadotropin-Releasing Hormone Agonist in Different Menstrual Cycles: A Retrospective Cohort Study. PeerJ (2019) 7: e6837. doi: $10.7717 /$ peerj.6837

6. Ren J, Sha A, Han D, Li P, Geng J, Ma C. Does Prolonged Pituitary DownRegulation With Gonadotropin-Releasing Hormone Agonist Improve the Live-Birth Rate in In Vitro Fertilization Treatment? Fertil Steril (2014) 102 (1):75-81. doi: 10.1016/j.fertnstert.2014.03.030

7. Bourgain C, Devroey P. The Endometrium in Stimulated Cycles for IVF. Hum Reprod Update (2003) 9(6):515-22. doi: 10.1093/humupd/dmg045

8. Shi Y, Sun Y, Hao C, Zhang H, Wei D, Zhang Y, et al. Transfer of Fresh Versus Frozen Embryos in Ovulatory Women. N Engl J Med (2018) 378 (2):126-36. doi: 10.1056/NEJMoa1705334

9. Ghobara T, Gelbaya TA, Ayeleke RO. Cycle Regimens for Frozen-Thawed Embryo Transfer. Cochrane Database Syst Rev (2017) 7:CD003414. doi: 10.1002/14651858.CD003414.pub3

10. Groenewoud ER, Cantineau AE, Kollen BJ, Macklon NS, Cohlen BJ. What Is the Optimal Means of Preparing the Endometrium in Frozen-Thawed Embryo Transfer Cycles? A Systematic Review and Meta-Analysis. Hum Reprod Update (2017) 23(2):255-61. doi: 10.1093/humupd/dmw046

11. Geng Y, Xun Y, Hu S, Lai Q, Jin L. GnRH Antagonist Versus Follicular-Phase Single-Dose GnRH Agonist Protocol in Patients of Normal Ovarian Responses During Controlled Ovarian Stimulation. Gynecol Endocrinol (2019) 35(4):309-13. doi: 10.1080/09513590.2018.1528221

12. Xu B, Geerts D, Hu S, Yue J, Li Z, Zhu G, et al. The Depot GnRH Agonist Protocol Improves the Live Birth Rate Per Fresh Embryo Transfer Cycle, But Not the Cumulative Live Birth Rate in Normal Responders: A Randomized Controlled Trial and Molecular Mechanism Study. Hum Reprod (2020) 35 (6):1306-18. doi: 10.1093/humrep/deaa086

13. Wang R, Lin S, Wang Y, Qian W, Zhou L. Comparisons of GnRH Antagonist Protocol Versus GnRH Agonist Long Protocol in Patients With Normal Ovarian Reserve: A Systematic Review and Meta-Analysis. PloS One (2017) 12 (4):e0175985. doi: 10.1371/journal.pone.0175985

14. Song J, Sun X, Qian K. Endometrial But Not Ovarian Response Is Associated With Clinical Outcomes and can be Improved by Prolonged Pituitary Downregulation in Patients With Thin and Medium Endometrium. Reprod Sci (2019) 26(11):1409-16. doi: 10.1177/1933719118816835

15. Mounce G, McVeigh E, Turner K, Child TJ. Randomized, Controlled Pilot Trial of Natural Versus Hormone Replacement Therapy Cycles in Frozen
Supervision: S-BC and Yun Zhou. Validation: S-BC and Y-HW. Writing - original draft: J-CL and L-YP. Writing review \& editing: S-BC. All authors contributed to the article and approved the submitted version.

\section{FUNDING}

This study was supported by Key Research and Development projects of JiangXi Province, China (NO: 20171BBG70010, 20192BBG70005).

Embryo Replacement In Vitro Fertilization. Fertil Steril (2015) 104(4):91520.el. doi: 10.1016/j.fertnstert.2015.07.1131

16. Yu S, Long H, Chang HY, Liu Y, Gao H, Zhu J, et al. New Application of Dydrogesterone as a Part of a Progestin-Primed Ovarian Stimulation Protocol for IVF: A Randomized Controlled Trial Including 516 First IVF/ICSI Cycles. Hum Reprod (2018) 33(2):229-37. doi: 10.1093/humrep/ $\operatorname{dex} 367$

17. Cummins JM, Breen TM, Harrison KL, Shaw JM, Wilson LM, Hennessey JF. A Formula for Scoring Human Embryo Growth Rates in In Vitro Fertilization: Its Value in Predicting Pregnancy and in Comparison With Visual Estimates of Embryo Quality. J In Vitro Fert Embryo Transf (1986) 3(5):284-95. doi: 10.1007/BF01133388

18. Trounson A, Mohr L. Human Pregnancy Following Cryopreservation, Thawing and Transfer of an Eight-Cell Embryo. Nature (1983) 305 (5936):707-9. doi: 10.1038/305707a0

19. De Neubourg D, Peeraer K, Debrock S, D’Hooghe T. Belgium Model of Coupling Reimbursement of ART Costs to Restriction in Number of Embryos Transferred. BMJ (2014) 348:g1559. doi: 10.1136/bmj.g1559

20. D'Angelo A, Amso NN. Embryo Freezing for Preventing Ovarian Hyperstimulation Syndrome: A Cochrane Review. Hum Reprod (2002) 17 (11):2787-94. doi: 10.1093/humrep/17.11.2787

21. Craciunas L, Gallos I, Chu J, Bourne T, Quenby S, Brosens JJ, et al. Conventional and Modern Markers of Endometrial Receptivity: A Systematic Review and Meta-Analysis. Hum Reprod Update (2019) 25 (2):202-23. doi: 10.1093/humupd/dmy044

22. He Z, Ma Y, Li L, Liu J, Yang H, Chen C, et al. Osteopontin and Integrin Alphavbeta3 Expression During the Implantation Window in IVF Patients With Elevated Serum Progesterone and Oestradiol Level. Geburtshilfe Frauenheilkd (2016) 76(6):709-17. doi: 10.1055/s-0041-111697

23. Cai J, Liu L, Zheng J, Zhang L, Jiang X, Li P, et al. Differential Response of AMH to GnRH Agonist Among Individuals: The Effect on Ovarian Stimulation Outcomes. J Assist Reprod Genet (2018) 35(3):467-73. doi: 10.1007/s10815-017-1095-Z

24. Martinez F, Clua E, Devesa M, Rodriguez I, Arroyo G, Gonzalez C, et al. Comparison of Starting Ovarian Stimulation on Day 2 Versus Day 15 of the Menstrual Cycle in the Same Oocyte Donor and Pregnancy Rates Among the Corresponding Recipients of Vitrified Oocytes. Fertil Steril (2014) 102 (5):1307-11. doi: 10.1016/j.fertnstert.2014.07.741

25. Bahceci M, Ulug U, Erden HF, Tosun S, Ciray N. Frozen-Thawed CleavageStage Embryo Transfer Cycles After Previous GnRH Agonist or Antagonist Stimulation. Reprod BioMed Online (2009) 18(1):67-72. doi: 10.1016/S14726483(10)60426-9

26. Lee JR, Choi YS, Jee BC, Ku SY, Suh CS, Kim KC, et al. Cryopreserved Blastocyst Transfer: Impact of Gonadotropin-Releasing Hormone Agonist Versus Antagonist in the Previous Oocyte Retrieval Cycles. Fertil Steril (2007) 88(5):1344-9. doi: 10.1016/j.fertnstert.2007.01.013

27. Eldar-Geva T, Zylber-Haran E, Babayof R, Halevy-Shalem T, Ben-Chetrit A, Tsafrir A, et al. Similar Outcome for Cryopreserved Embryo Transfer Following GnRH-Antagonist/GnRH-Agonist, GnRH-Antagonist/HCG or Long Protocol Ovarian Stimulation. Reprod BioMed Online (2007) 14 (2):148-54. doi: 10.1016/S1472-6483(10)60781-X 
28. Blockeel C, Campbell A, Coticchio G, Esler J, Garcia-Velasco JA, Santulli P, et al. Should We Still Perform Fresh Embryo Transfers in ART? Hum Reprod (2019) 34(12):2319-29. doi: 10.1093/humrep/dez233

Conflict of Interest: The authors declare that the research was conducted in the absence of any commercial or financial relationships that could be construed as a potential conflict of interest.

Publisher's Note: All claims expressed in this article are solely those of the authors and do not necessarily represent those of their affiliated organizations, or those of the publisher, the editors and the reviewers. Any product that may be evaluated in this article, or claim that may be made by its manufacturer, is not guaranteed or endorsed by the publisher.

Copyright $\odot 2021$ Li, Wang, Peng, Zhou and Chao. This is an open-access article distributed under the terms of the Creative Commons Attribution License (CC BY). The use, distribution or reproduction in other forums is permitted, provided the original author(s) and the copyright owner(s) are credited and that the original publication in this journal is cited, in accordance with accepted academic practice. No use, distribution or reproduction is permitted which does not comply with these terms. 